\title{
Researches on Welder's Pneumoconiosis Risk Assessment Methods Based on Logistic Regression
}

\author{
Deyin Huang ${ }^{1}$, Minyan $\mathrm{Li}^{1}$, Mao Liu ${ }^{2}$ \\ ${ }^{1}$ Institute of Occupational Health, Tianjin Bohai Chemical Industry Group Co. Ltd, Tianjin, 300051, China \\ ${ }^{2}$ Centre for Urban Public Safety Research, Nankai University, Tianjin, 300071, China
}

\section{基于 logistic 回归模型的电焊工尘肺风险评价方法研究 \\ 黄德寅 ${ }^{1}$, 李敏嫣 ${ }^{1}$, 刘茂 ${ }^{2}$ \\ ${ }^{1}$ 天津渤海化工集团有限责任公司劳动卫生研究所, 天津 300051 , 中国 \\ 2 南开大学城市公共安全研究中心, 天津 300071 , 中国}

\begin{abstract}
Objective To research the risk assessment method of welder's pneumoconiosis caused by welding fume exposure, predict the incidence of welder's pneumoconiosis, and provide technical basis for occupational risk management. Method Welders of a large-scale agricultural machinery processing workshop were chosen as objectives. The incidence of welder's pneumoconiosis was predicted by logistic non-linear regression model using the concentrations of the welding fume that the welders exposed to and their exposure durations. Furthermore, the relationship between the incidence probability of welder's pneumoconiosis and exposure situation was found by using logistic regression model, and the incidence probability distribution was evaluated by Monte-Carlo simulation. Results It was found that if these welders exposed to the welding fume of the current concentration for 5 or 10 years, the risk of welder's pneumoconiosis would be between $10^{-3}$ and $10^{-2}$, and for 20 or 30 years, it would be between $10^{-2}$ and $10^{-1}$. Assuming that they exposed to welding fume of this concentration for 30 years, the risk would exceed the acceptable risk level and measures should be taken. Conclusion In this study, a practical quantitative approach was proposed to assess the risk of welder's pneumoconiosis caused by welding fume exposure. Based on the results, it showed that as the risk management target, the concentration and exposure
\end{abstract}

duration should be well controlled to reduce the risk of welder's pneumoconiosis. This method could be applied in evaluations of occupational disease hazard in construction projects and help to control and manage the risk of welder's pneumoconiosis.

Keywords: Welding fume; Welders' pneumoconiosis; Risk assessment; Logistic regression model

\section{摘要}

目的 研究电焊工接触电焊烟尘所致电焊工尘肺风险 分析方法, 预测电焊工尘肺的风险概率, 为实施风险 管理提供技术依据。方法 选取某大型农用机械加工 电焊车间作为研究对象。采用 logistic 回归模型的非 线性回归分析, 通过接尘浓度以及接尘工龄预测电焊 工尘肺发病概率; 采用计算机 $\mathrm{R}$ 语言进行接尘工龄、 接尘浓度与发病概率之间关系的风险表征结果查表 实用性研究; 采用 logistic 回归模型-蒙特卡洛模拟方 法分析职业暴露与电焊工尘肺发病概率的分布规律 研究。结果 焊接作业场所电焊工的暴露于目前电焊 烟尘浓度 5 年和 10 年发生电焊工尘肺的风险在 $10^{-3} \sim 10^{-2}$, 暴露 20 年和 30 年发生电焊工尘肺的风险 在 $10^{-2} \sim 10^{-1}$ 。按职业生涯 30 年工龄计, 发生电焊工 尘肺的风险高于可接受风险水平, 需要采取措施降低 风险。结论 本研究建立电焊烟尘致电焊工尘肺的定 量风险评价方法, 以电焊工接尘工龄、电焊烟尘暴露 浓度与电焊工尘肺的风险概率作为风险目标, 实用性

基金项目：天津市科技支撑计划重点项目（13ZCZDSY02300）

黄德寅 (1962-) , 女, 主任医师, 主要研究方向为职业卫生与风险分析 
Risk Analysis and Crisis Response in Big Data Era (RAC-16)

强, 得以实现在职业病危害评价的实际应用, 为电焊 工尘肺的预防控制提供了理论依据。

关键词: 电焊烟尘; 电焊工尘肺; 风险评价; logistic 回归模型

\section{1. 引言}

电焊烟尘是指在焊接作业时, 由于高温使焊药、焊条 和被焊接材料熔化蒸发, 逸散在空气中氧化冷凝而形 成的颗粒极细的气溶胶 (直径小于 $5 \mu \mathrm{m}$ )。由于电焊 烟尘的粒径很小, 属呼吸性粉尘。电焊工尘肺是操作 者由于长期吸入高浓度的电焊烟尘, 引起肺组织弥漫 性纤维病变的疾病, 是我国法定职业病之一 [1] [2], 是一种以氧化铁为主、同时混合有氧化锰、二氧化硅 等粉尘引起的一种混合性尘肺。以往的流行病学资料 显示, 电焊工尘肺发病工龄多在 7 23 年, 平均工龄 20 年。近年来由于行业结构特点的变化, 电焊工尘 肺在天津市及某些地区呈现发病人数逐年上升的趋 势, 甚至成为报告尘肺病的主要病种, 且呈现发病年 龄轻、接尘工龄短的特点, 电焊工尘肺的防治工作刻 不容缓[3]。

针对我国电焊工尘肺的职业病危害形势, 我们采 用健康风险评价四步法对电焊烟尘职业暴露下作业 工人发生电焊工尘肺的风险进行定量评价方法的研 究, 建立了基于 logistic 回归模型的电焊工尘肺风险 评价方法。本文将结合某大型农用机械加工车间的实 例应用进行电焊烟尘根据目前国际上普遍采用的 EPA 健康风险评价四步法的步骤进行电焊烟尘职业 暴露风险评价, 即危害辨识、暴露评价、剂量-反应 评价和风险表征等 [4] [5]。电焊烟尘职业暴露风险评 价方法程序与内容如下:

（1）危害辨识: 通过对工作场所的电焊烟尘职 业暴露及潜在风险进行辨识, 收集相关资料, 包括职 业史、暴露人群数量、性别、年龄分布、暴露方式、 暴露时间、工人防护条件等, 电焊烟尘健康影响, 人 群流行病学数据等。

（2）暴露评价：是基于电焊烟尘浓度进行现场 检测的数据, 对此进行统计分布检验, 得到接尘浓度 的概率分布; 采用统计学软件 SPSS 19.0.0 的 Shapiro-Wilk (W 检验) 进行改造期间电焊烟尘现场 检测 TWA 浓度（以时间为权数规定的 $8 \mathrm{~h}$ 工作日的 平均接触浓度) 的正态性检验, 得到改造期间外暴露 浓度的分布。

（3）剂量-反应评价: 选择 logistic 模型作为电 焊工尘肺的剂量-反应模型, 利用人群流行病学资料
确定模型参数, 得到用于评价电焊烟尘暴露致电焊工 尘肺的剂量-反应模型; 采用统计学软件 SPSS 19.0.0 的非线性回归拟合电焊烟尘职业暴露导致电焊工尘 肺的剂量-反应模型参数。

（4）风险表征: 将经统计处理后的现场电焊烟 尘检测浓度代入剂量-反应模型直接计算, 求解不同 工龄发生电焊工尘肺的风险, 对电焊烟尘暴露人群在 此环境下长期工作所导致电焊工尘肺的风险进行表 征, 并对风险评价过程中的不确定性采用 Crystal Ball 软件进行 Monte Carlo 模拟; 采用计算机 R 语言, 接 尘浓度、接尘工龄与电焊工尘肺发病率之间关系表。

\section{2. 电焊烟尘职业暴露风险评价方法技术路线}

具体技术路线如图 1 所示。

\section{3. 实例应用研究}

\section{1. 研究对象工艺简介}

选取某大型农用机械加工电焊车间作为研究对象。某 大型农用机械制造企业主要生产 6 吨以下四轮驱动 装载机、30 吨以下履带式挖掘机和四轮挖掘机, 生 产规模为年产 3000 台。电焊车间焊接工序采用二氧 化碳气体保护焊半自动焊接工艺, 即焊丝送进靠机械 自动进行, 由焊工手持焊炬进行焊接操作。

\section{2. 焊接车间职业卫生调查}

焊接工序分为装载机焊接区域和驾驶室焊接区域, 装 载机焊接区域设有 10 个焊接工位, 驾驶室焊接区域 设有 8 个焊接工位。生产厂房内设有全面通风设施, 为作业工人配备焊接头盔、防尘口罩、防护手套等。 每个焊接工位操作工为固定岗作业, 驾驶室焊接操作 工为三班制, 每班 8 小时; 装载机焊接操作工为单班 制, 每班 8 小时。班制及劳动定员见表 1 。

\section{3. 工作场所电焊烟尘暴露水平测定}

主要依据 GBZ159-2004《工作场所空气中有害物质监 测的采样规范》、GBZ/T192.1-2007《工作场所空气中 粉尘测定第 1 部分: 总粉尘浓度》进行现场采样及实 验室检测。由于电焊车间工作场所空气中电焊烟尘浓 度超过职业接触限值, 该企业为控制电焊烟尘对工作 环境的污染, 共进行了 3 次通风除尘系统改造, 在通 风系统改造前及改造后共进行了四次个体采样及定 点采样。个体采样 TWA 浓度检测值见图 2 。 
Risk Analysis and Crisis Response in Big Data Era (RAC-16)

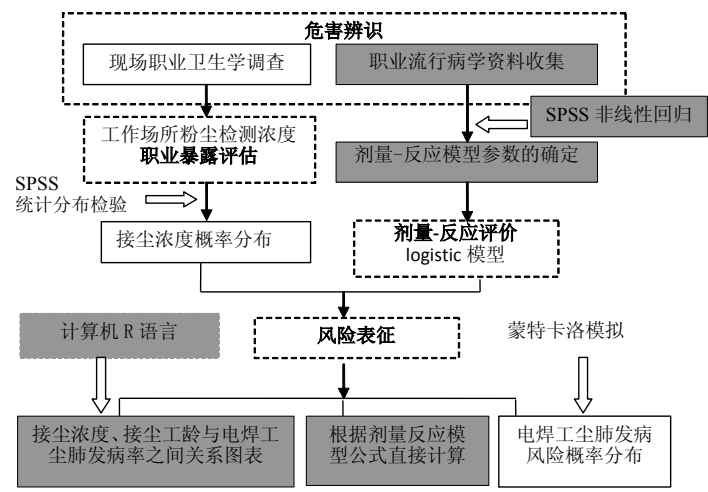

图 1. 电焊烟尘职业病风险评价方法技术路线

表 1. 生产班制及劳动定员

\begin{tabular}{|c|c|c|c|c|}
\hline 岗位 & 生产班制 & 每班定员 & 总定员 & 操作方式及每班操作时间 \\
\hline 装载机焊工 & 单班制, $8 \mathrm{~h} /$ 班 & 12 & 12 & $\begin{array}{l}\text { 半自动化 } \mathrm{CO}_{2} \text { 保护焊, 每班累计操作时 } \\
\text { 间 } 8 \text { 小时 }\end{array}$ \\
\hline 驾驶室焊工 & 三班制, $8 \mathrm{~h} /$ 班 & 6 & 18 & $\begin{array}{l}\text { 半自动化 } \mathrm{CO}_{2} \text { 保护焊, 每班累计操作时 } \\
\text { 间 } 8 \text { 小时 }\end{array}$ \\
\hline
\end{tabular}

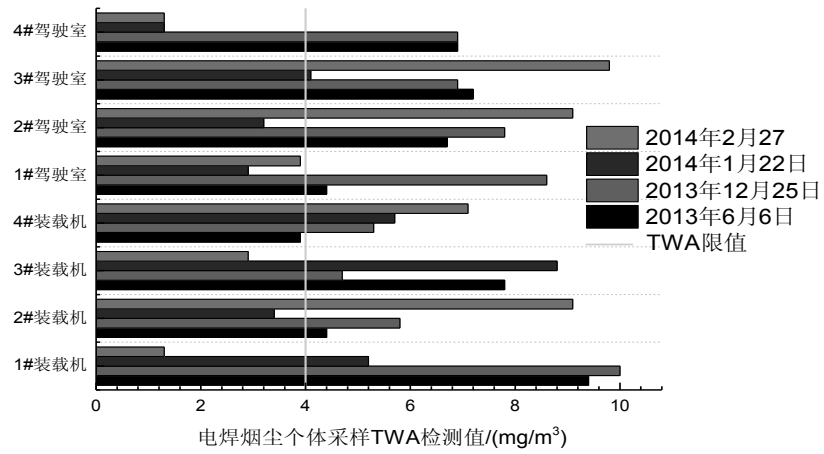

图 2. 电焊烟尘个体采样 TWA 检测值

对四次检测结果采用 SPSS 19.0.0 进行随机区组 设计资料的方差分析。经检验, $P$ 值 $=0.495>0.05$, 可 以认为检测时间 (即通风改造效果) 对装载机焊接工 位电焊烟尘浓度的效应不显著, 即通风改造无显著效 果。对该车间焊接工位的电焊烟尘三次改造检测结果 采用 Shapiro-Wilk ( $\mathrm{W}$ 检验), 所得结果如表 2 所示。 经检验, $P=0.331>0.05$, 可以认为电焊烟尘浓度服从 均值为 5.55 , 标准差为 2.714 的正态分布。
表 2. 焊接工位电焊烟尘浓度的正态性检验

\begin{tabular}{ll}
\hline 统计量 & 结果 \\
\hline 均值 & 5.550 \\
\hline 标准差 & 2.714 \\
\hline $\mathrm{df}$ & 22 \\
\hline Shapiro-Wilk Sig. & 0.331 \\
\hline
\end{tabular}


Risk Analysis and Crisis Response in Big Data Era (RAC-16)

\section{4. 基于 logistic 模型的电焊工尘肺剂量-反应关系}

\section{(1) Logistic 回归模型}

本文选择 logistic 模型作为电焊工尘肺的剂量-反应模 型。Logistic 回归模型作为一种统计方法, 可以客观 真实的呈现危险因素与患病风险的内在联系, 并将该 关系数量化。

logistic 模型基本原理: 采用一系列案例数据对 logistic 回归模型进行拟合; 再则, 采用该模型呈现 某些自变量和因变量取特定值之概率的关系。与此同 时, 控制特定自变量之外的一切自变量, 随之反映该 特定自变量对因变量取某特定值之概率的决定性作 用的大小。将影响电焊工尘肺的危害因素作为自变 量, 电焊工尘肺患病率作为因变量, 进行多因素 logistic 回归分析, 拟合 logistic 回归模型。下式表示 某病发病概率 $P_{i}$ 与年日接尘浓度 $a$ 、接尘工龄 $b$ 之间
的关系:

$$
P=\frac{\exp \left[\beta_{i 0}+\beta_{i 1} \ln a b\right]}{1+\exp \left[\beta_{i 0}+\beta_{i 1} \ln a b\right]}
$$

式中:

$P_{i}$ 为不同接尘浓度、接尘工龄的电焊工尘肺发病率; $\beta_{i 0}$ 为 logistic 回归分析的常数项;

$\beta_{i 1}$ 为 logistic 回归分析的回归系数; $a$ 为接尘浓度 $\left(\mathrm{mg} / \mathrm{m}^{3}\right)$;

$b$ 为接尘工龄 (年)。

\section{(2) logistic 模型的参数拟合}

收集公开发表的国内典型行业电焊工尘肺流行病学 调查资料, 根据各调查实例中的电焊烟尘暴露水平和 电焊工尘肺发病率数据, 拟合 logistic 模型中的参数 值。本文主要收集并采用了以下电焊工尘肺的流行病 学调查资料 [5-20], 见表 3 。

表 3. 电焊工尘肺人群流行病学调查结果

\begin{tabular}{|c|c|c|c|c|c|c|}
\hline 城市 & 行业 & $\begin{array}{l}\text { 接尘浓度 } \\
\left(\mathrm{mg} / \mathrm{m}^{3}\right)\end{array}$ & $\begin{array}{l}\text { 接尘工龄 } \\
\text { (年) }\end{array}$ & 受检人数 & 患病人数 & 患病率 \\
\hline- & 石油勘探开发 & 3.7570 & 26.50 & 356 & 2 & 0.0056 \\
\hline- & & 36.4500 & 16.70 & 1160 & 8 & 0.0069 \\
\hline 上海 & 汽轮机厂 & 2.7000 & 26.00 & 59 & 1 & 0.0170 \\
\hline 上海 & 汽轮机厂 & 5.1900 & 12.00 & 49 & 1 & 0.0200 \\
\hline- & 钢构厂 & 11.5071 & 23.00 & 108 & 6 & 0.0556 \\
\hline- & - & 30.3800 & 23.15 & 660 & 87 & 0.1318 \\
\hline 大连 & 造船厂 & 80.1560 & 32.00 & 204 & 114 & 0.5588 \\
\hline- & 集装箱制造 & 25.0000 & 10.60 & 220 & 19 & 0.0860 \\
\hline 齐齐哈尔 & 车辆厂 & 16.4433 & 23.15 & 660 & 87 & 0.1318 \\
\hline 北京 & 铁路机务系统 & 7.9750 & 18.00 & 105 & 9 & 0.0857 \\
\hline 镇江 & - & 4.7000 & 20.00 & 1756 & 50 & 0.0285 \\
\hline- & 机械厂 & 1.9400 & 14.20 & 110 & 5 & 0.0455 \\
\hline 广东 & 集装箱制造 & 5.9464 & 8.00 & 717 & 15 & 0.0209 \\
\hline- & - & 4.8600 & 18.50 & 162 & 1 & 0.0062 \\
\hline- & 集装箱制造 & 7.4351 & 8.22 & 1561 & 13 & 0.0083 \\
\hline- & - & 2.6100 & 10.97 & 86 & 0 & 0.0000 \\
\hline
\end{tabular}

根据表 3 中电焊烟尘职业暴露浓度与电焊烟尘 发病率间的剂量-反应关系, 利用 SPSS 19.0.0 的 logistic 非线性回归对收集的电焊工尘肺流行病学调 查数据进行拟合。得到 logistic 模型中的参数值 $\beta_{i 0}=-11.814 、 \beta_{i I}=1.526, R^{2}=0.897$ 。通过检验, 可以 认为接尘浓度与接尘工龄的乘积, 即累积接尘量与发 病率相关。则电焊工尘肺的剂量-反应模型为:

$$
P=\frac{\exp [-11.814+1.526 \ln a b]}{1+\exp [-11.814+1.526 \ln a b]}
$$
式中: $a$ 为接尘浓度, 即 $8 \mathrm{~h}$ 时间加权平均浓度 $\left(\mathrm{mg} / \mathrm{m}^{3}\right)$; $b$ 为接尘工龄 (年)。 
Risk Analysis and Crisis Response in Big Data Era (RAC-16)

\section{5. 风险表征与蒙特卡洛模拟}

表 5. 预测不同接尘工龄各分位数对应发生电焊工尘肺的风险

\section{(1) 直接计算}

\begin{tabular}{lllll}
\hline 分位数 & 5 年 & 10 年 & 20 年 & 30 年 \\
\hline $0 \%$ & $5.41 \times 10^{-3}$ & $1.76 \times 10^{-2}$ & $4.15 \times 10^{-2}$ & $9.82 \times 10^{-2}$ \\
\hline $10 \%$ & $2.48 \times 10^{-3}$ & $7.22 \times 10^{-3}$ & $2.06 \times 10^{-2}$ & $3.69 \times 10^{-2}$ \\
\hline $20 \%$ & $2.01 \times 10^{-3}$ & $5.88 \times 10^{-3}$ & $1.66 \times 10^{-2}$ & $3.00 \times 10^{-2}$ \\
\hline $30 \%$ & $1.68 \times 10^{-3}$ & $4.92 \times 10^{-3}$ & $1.40 \times 10^{-2}$ & $2.53 \times 10^{-2}$ \\
\hline $40 \%$ & $1.43 \times 10^{-3}$ & $4.15 \times 10^{-3}$ & $1.18 \times 10^{-2}$ & $2.14 \times 10^{-2}$ \\
\hline $50 \%$ & $1.21 \times 10^{-3}$ & $3.51 \times 10^{-3}$ & $9.96 \times 10^{-3}$ & $1.80 \times 10^{-2}$ \\
\hline $60 \%$ & $1.00 \times 10^{-3}$ & $2.88 \times 10^{-3}$ & $8.25 \times 10^{-3}$ & $1.50 \times 10^{-2}$ \\
\hline $70 \%$ & $7.75 \times 10^{-4}$ & $2.27 \times 10^{-3}$ & $6.60 \times 10^{-3}$ & $1.18 \times 10^{-2}$ \\
\hline $80 \%$ & $5.65 \times 10^{-4}$ & $1.61 \times 10^{-3}$ & $4.75 \times 10^{-3}$ & $8.64 \times 10^{-3}$ \\
\hline $90 \%$ & $3.18 \times 10^{-4}$ & $8.71 \times 10^{-4}$ & $2.72 \times 10^{-3}$ & $4.94 \times 10^{-3}$ \\
\hline $100 \%$ & 0 & 0 & 0 & 0 \\
\hline & & & &
\end{tabular}

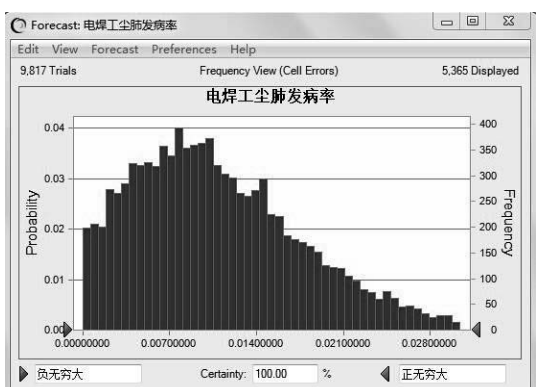

图 3. 电焊工 20 年接尘工龄发生电焊工尘肺风险的概率分布

表 4. 预测不同接尘工龄发生电焊工尘肺的风险统计分析结果

\begin{tabular}{lllll}
\hline 统计量 & 5 年 & 10 年 & 20 年 & 30 年 \\
\hline 均值 & $1.33 \times 10^{-3}$ & $3.84 \times 10^{-3}$ & $1.10 \times 10^{-2}$ & $1.98 \times 10^{-2}$ \\
\hline 中位数 & $1.21 \times 10^{-3}$ & $3.51 \times 10^{-3}$ & $9.96 \times 10^{-3}$ & $1.80 \times 10^{-2}$ \\
\hline 标准差 & $8.49 \times 10^{-4}$ & $2.46 \times 10^{-3}$ & $6.92 \times 10^{-3}$ & $1.25 \times 10^{-2}$ \\
\hline 最小值 & 0 & 0 & 0 & 0 \\
\hline 最大值 & $5.41 \times 10^{-3}$ & $1.76 \times 10^{-2}$ & $4.15 \times 10^{-2}$ & $9.82 \times 10^{-2}$ \\
\hline
\end{tabular}

（2）基于计算机 $\mathbf{R}$ 语言的接尘浓度、接尘工龄与发 病率关系表

为了提高本研究风险评价方法的实用性, 方便计算电 焊烟尘职业暴露的风险, 利用 $\mathrm{R}$ 语言给出 8 小时时 间加权平均接尘浓度、接尘工龄与电焊工尘肺发病率 之间关系的速查表, 即 $0.1-10.0 \mathrm{mg} / \mathrm{m}^{3}$ 接尘浓度和 5 年、 10 年、 20 年和 30 年接尘工龄对应的电焊工尘肺 发病率可以通过查表查得。以电焊工 20 年接尘工龄 为例, 暴露于不同 TWA 接尘浓度下电焊工尘肺发病 风险预测见表 6 。

\section{6. 风险控制对策}

由风险表征结果可以看出, 假设该电焊车间焊接作业 场所电焊工人不佩戴防尘口罩, 暴露于该作业场所的 电焊烟尘浓度 5 年和 10 年发生电焊工尘肺的风险在 $10^{-3} \sim 10^{-2}$, 暴露 20 年和 30 年发生电焊工尘肺的风险 在 $10^{-2} \sim 10^{-1}$ 。按职业生涯 30 年工龄计, 发生电焊工 尘肺的风险高于可接受风险水平, 需要采取措施降低 风险。

针对电焊烟尘的职业病危害高风险, 根据风险优 先权应首选工程技术控制措施, 结合历次电焊烟尘的 检测结果, 分析目前采取的通风形式不能起到将电焊 烟尘全面排出的效果, 应通过合理有效的全面及局部 通风的方式进行控制; 采取严格的职业卫生管理措施 加强焊接作业工人的个人防护, 为工人配备防尘口 罩, 并指导和监督工人正确佩戴; 加强对作业工人进 行呼吸防护用品的使用及维护的培训和教育, 要求操 作工严格遵守操作规程; 加强防护设施的日常维护和 检修, 使其保持正常的运行状态以及良好的通风除尘 效果; 经常性地进行职业健康检查, 并且需要对电焊 烟尘的高风险进行日常监测等组织管理措施控制并 降低风险。

\section{4. 结论与讨论}

目前, 国内外的学者主要利用 logistic 回归方法进行 篮选影响电焊工尘肺的危险因素和预测发病趋势的 研究[21], 很少采用 logistic 回归方法预测尘肺发病 率与接尘浓度、接尘时间之间的剂量-反应关系。本 文建立的电焊烟尘职业暴露风险评价方法以常规流 行病学资料为基础, 运用 logistic 回归模型的非线性 
Risk Analysis and Crisis Response in Big Data Era (RAC-16)

表 6.20 年接尘工龄暴露于不同 TWA 接尘浓度下电焊工尘肺发病风险预测

\begin{tabular}{|c|c|c|c|c|c|c|c|c|c|}
\hline $\begin{array}{l}\text { 接尘浓度 } \\
\left(\mathrm{mg} / \mathrm{m}^{3}\right)\end{array}$ & 风险值 & $\begin{array}{l}\text { 接尘浓度 } \\
\left(\mathrm{mg} / \mathrm{m}^{3}\right)\end{array}$ & 风险值 & $\begin{array}{l}\text { 接尘浓度 } \\
\left(\mathrm{mg} / \mathbf{m}^{3}\right)\end{array}$ & 风险值 & $\begin{array}{l}\text { 接尘浓度 } \\
\left(\mathrm{mg} / \mathbf{m}^{3}\right)\end{array}$ & 风险 & $\begin{array}{l}\text { 接尘浓度 } \\
\left(\mathrm{mg} / \mathbf{m}^{3}\right)\end{array}$ & 风险值 \\
\hline 0.1 & $2.13 \times 10^{-5}$ & 2.1 & $2.21 \times 10^{-3}$ & 4.1 & $6.12 \times 10^{-3}$ & 6.1 & $1.12 \times 10^{-2}$ & 8.1 & $1.71 \times 10^{-2}$ \\
\hline 0.2 & $6.14 \times 10^{-5}$ & 2.2 & $2.38 \times 10^{-3}$ & 4.2 & $6.35 \times 10^{-3}$ & 6.2 & $1.14 \times 10^{-2}$ & 8.2 & $1.74 \times 10^{-2}$ \\
\hline 0.3 & $1.14 \times 10^{-4}$ & 2.3 & $2.54 \times 10^{-3}$ & 4.3 & $6.58 \times 10^{-3}$ & 6.3 & $1.17 \times 10^{-2}$ & 8.3 & $1.78 \times 10^{-2}$ \\
\hline 0.4 & $1.77 \times 10^{-4}$ & 2.4 & $2.71 \times 10^{-3}$ & 4.4 & $6.82 \times 10^{-3}$ & 6.4 & $1.20 \times 10^{-2}$ & 8.4 & $1.81 \times 10^{-2}$ \\
\hline 0.5 & $2.48 \times 10^{-4}$ & 2.5 & $2.89 \times 10^{-3}$ & 4.5 & $7.05 \times 10^{-3}$ & 6.5 & $1.23 \times 10^{-2}$ & 8.5 & $1.84 \times 10^{-2}$ \\
\hline 0.6 & $3.28 \times 10^{-4}$ & 2.6 & $3.07 \times 10^{-3}$ & 4.6 & $7.29 \times 10^{-3}$ & 6.6 & $1.26 \times 10^{-2}$ & 8.6 & $1.87 \times 10^{-2}$ \\
\hline 0.7 & $4.15 \times 10^{-4}$ & 2.7 & $3.25 \times 10^{-3}$ & 4.7 & $7.53 \times 10^{-3}$ & 6.7 & $1.29 \times 10^{-2}$ & 8.7 & $1.91 \times 10^{-2}$ \\
\hline 0.8 & $5.09 \times 10^{-4}$ & 2.8 & $3.43 \times 10^{-3}$ & 4.8 & $7.78 \times 10^{-3}$ & 6.8 & $1.32 \times 10^{-2}$ & 8.8 & $1.94 \times 10^{-2}$ \\
\hline 0.9 & $6.09 \times 10^{-4}$ & 2.9 & $3.62 \times 10^{-3}$ & 4.9 & $8.02 \times 10^{-3}$ & 6.9 & $1.35 \times 10^{-2}$ & 8.9 & $1.97 \times 10^{-2}$ \\
\hline 1.0 & $7.15 \times 10^{-4}$ & 3.0 & $3.81 \times 10^{-3}$ & 5.0 & $8.27 \times 10^{-3}$ & 7.0 & $1.37 \times 10^{-2}$ & 9.0 & $2.00 \times 10^{-2}$ \\
\hline 1.1 & $8.27 \times 10^{-4}$ & 3.1 & $4.01 \times 10^{-3}$ & 5.1 & $8.52 \times 10^{-3}$ & 7.1 & $1.40 \times 10^{-2}$ & 9.1 & $2.04 \times 10^{-2}$ \\
\hline 1.2 & $9.44 \times 10^{-4}$ & 3.2 & $4.20 \times 10^{-3}$ & 5.2 & $8.78 \times 10^{-3}$ & 7.2 & $1.43 \times 10^{-2}$ & 9.2 & $2.07 \times 10^{-2}$ \\
\hline 1.3 & $1.07 \times 10^{-3}$ & 3.3 & $4.41 \times 10^{-3}$ & 5.3 & $9.03 \times 10^{-3}$ & 7.3 & $1.46 \times 10^{-2}$ & 9.3 & $2.11 \times 10^{-2}$ \\
\hline 1.4 & $1.19 \times 10^{-3}$ & 3.4 & $4.61 \times 10^{-3}$ & 5.4 & $9.29 \times 10^{-3}$ & 7.4 & $1.49 \times 10^{-2}$ & 9.4 & $2.14 \times 10^{-2}$ \\
\hline 1.5 & $1.33 \times 10^{-3}$ & 3.5 & $4.82 \times 10^{-3}$ & 5.5 & $9.56 \times 10^{-3}$ & 7.5 & $1.53 \times 10^{-2}$ & 9.5 & $2.17 \times 10^{-2}$ \\
\hline 1.6 & $1.46 \times 10^{-3}$ & 3.6 & $5.03 \times 10^{-3}$ & 5.6 & $9.82 \times 10^{-3}$ & 7.6 & $1.56 \times 10^{-2}$ & 9.6 & $2.21 \times 10^{-2}$ \\
\hline 1.7 & $1.61 \times 10^{-3}$ & 3.7 & $5.24 \times 10^{-3}$ & 5.7 & $1.01 \times 10^{-2}$ & 7.7 & $1.59 \times 10^{-2}$ & 9.7 & $2.24 \times 10^{-2}$ \\
\hline 1.8 & $1.75 \times 10^{-3}$ & 3.8 & $5.46 \times 10^{-3}$ & 5.8 & $1.04 \times 10^{-2}$ & 7.8 & $1.62 \times 10^{-2}$ & 9.8 & $2.28 \times 10^{-2}$ \\
\hline 1.9 & $1.90 \times 10^{-3}$ & 3.9 & $5.68 \times 10^{-3}$ & 5.9 & $1.06 \times 10^{-2}$ & 7.9 & $1.65 \times 10^{-2}$ & 9.9 & $2.31 \times 10^{-2}$ \\
\hline 2.0 & $2.06 \times 10^{-3}$ & 4.0 & $5.90 \times 10^{-3}$ & 6.0 & $1.09 \times 10^{-2}$ & 8.0 & $1.68 \times 10^{-2}$ & 10.0 & $2.35 \times 10^{-2}$ \\
\hline
\end{tabular}

回归分析, 通过导致电焊工尘肺的主要因素接尘浓度 以及接尘工龄, 预测电焊工尘肺发病概率, 对电焊作 业场所职业病危害进行风险分析; 针对电焊作业职业 暴露与电焊工尘肺风险的不确定性, 采用 logistic 回 归模型-蒙特卡洛模拟方法分析暴露与电焊工尘肺发 病概率的分布规律; 风险表征采用计算机 $\mathrm{R}$ 语言进 行接尘工龄、接尘浓度与发病概率之间关系的可视化 研究, 并进行风险表征结果速查表实用性研究, 方便 职业卫生工作者使用查阅, 使得研究成果有很强的实 用性和可操作性。

通过实例应用证明本文建立的电焊烟尘职业暴 露评估与风险分析技术, 可以在建设项目职业病危害 评价工作中进行应用, 可以满足职业卫生评价对于评 价方法的需求。针对建设项目正常生产运行过程可能 造成劳动者发生电焊工尘肺的风险, 该方法可对焊接 车间的电焊烟尘职业暴露过程、可能发生职业病危害 后果及危害程度进行定量风险分析, 并进一步分析电 焊烟尘致电焊工尘肺等职业病危害潜在风险及防范 措施, 从预防和控制职业病危害的角度, 在工程技术、 健康监护、组织管理等方面针对性的提出风险控制措 施的建议, 为企业实施风险管理提供技术依据, 对保
障焊接车间电焊工的职业健康有重要意义。但电焊烟 尘职业暴露风险分析技术的研究需要在今后进一步 进行内暴露剂量反应评价的研究及对职业高危人群 电焊工尘肺的预警预测技术的研究。

\section{Acknowledgements}

This study was supported by Municipal Key Science and technology support project of Tianjin (No. 13ZCZDSY02300).

\section{致谢}

本研究得到了天津市科技支撑计划重点项目 (13ZCZDSY02300)的资助。

\section{参考文献}

[1] 陆春花, 朱宝立, 杨继红, 等. 某市电焊工尘肺监测 哨点电焊烟尘水平及接尘工人健康状况.中华劳 动卫生职业病杂志,2013,31(11):847-848.

[2] 周浩, 吴琳, 肖吕武. 电焊烟尘对接触工人肺功能 的影响.中华劳动卫生职业病杂志, 2014, 32(4): 
Risk Analysis and Crisis Response in Big Data Era (RAC-16)

287-288.

[3] 王莉萍.上海市金山区企业电焊烟尘的职业危害 状况.职业与健康, 2012(14):1674-1678.

[4] Qian Zhang, Deyin Huang, Mao Liu. Interna Exposure Simulation Based on Exposure Related Dose Estimating Model. Journal of Risk Analysis and Crisis Response. 2013, 3(4):175-184.

[5] Minyan Li, Deyin Huang,,Mao Liu. MCSimBased Occupational Health Risk Assessment on Benzene. Journal of Risk Analysis and Crisis Response, 2013, 3(1-4):135-145.

[6] 段建军.某石油勘探开发企业电焊工尘肺发病回 顾性分析.中国职业医学,2012,39(4):315-316。

[7] 王新.电焊工 1160 名健康状况分析.职业与健 康,2003,4:9-10。

[8] 陈月琴,张允睿,陆志英.上海汽轮机厂焊工尘肺 调查.劳动医学, 1996,3:27-29.

[9] 薛忠丽,孙玉欣.某钢构厂装配车间 6 例电焊工尘 肺调查与分析.工业卫生与职业病，2011，05: 318-319.

[10] 邹昌淇,邢康吉, 宁汉孙. 电焊工尘肺发病情况调 查.中华劳动卫生职业病杂志,1990,01:65-66

[11] 侯瑞罕,李正修,王永顺.大连造船厂 204名电焊工 的尘肺发展情况.中华劳动卫生职业病杂志, 1996, $1: 44-45$.

[12] 彭文彬.某集装箱制造公司电焊工尘肺高发原因
的调查分析.职业与健康,2006,1:19-20。

[13] 宁汉孙. 电焊工与电焊工尘肺的肺功能研究. 铁道 劳动安全卫生与环保,1988,3: 17-19.

[14] 李理. 电焊作业场所焊尘浓度的预测. 铁道劳动安 全卫生与环保, 1989,1:35-37

[15] 姜方平. 镇江市电焊作业工人健康状况调查. 职业 与健康,2009,17:1818-1820.

[16] 吴全兰, 许希海, 丁新平. 某机械厂电焊烟尘危害 调查.中国职业医学,2009,4:359-360.

[17] 丘创逸,张东辉,陈建雄. 某地集装箱制造企业电 焊工尘肺的流行病学调查. 中华劳动卫生职业病 杂志, 2009,27,4:215-217.

[18] 龙双才. 结构钢焊条对电焊作业人员职业病危害 调查分析.实用预防医学,2006,3:685-686.

[19] 曾林陵,林旭华,丘创逸. 某集装箱制造企业职业. 病危害现状调查分析.中国职业医学, 2006, 5:351-353

[20] 杨晓军,付远钊, 刘涁. 某基地电焊作业者呼吸系 统健康状况分析.中国航天科工集团公司.第二十 届航天医学年会暨第三届航天护理年会论文汇 编.中国航天科工集团公司,2004:2.

[21] 刘宝龙,樊晶光,陈胜.Logistic 回归模型在尘肺发 病预测与控制中的应用研究. 中国安全科学学 报,2001,11(1),40-43. 